\title{
Memorial Pere Alberch. "The Cradle of Evo-Devo". Valencia 21-24 Mayo de 2008
}

\author{
L. Boto ${ }^{1}$
}

Durante los días 21 a 24 de Mayo se ha celebrado en Valencia, organizado por el Instituto Cavanilles y bajo el título de "The cradle of Evo-Devo", un simposio internacional en homenaje a Pere Alberch Vie (Badalona 1954-Madrid 1998) en el décimo aniversario de su prematuro fallecimiento. Pere Alberch fue Profesor de Investigación del CSIC, director del Museo Nacional de Ciencias Naturales durante los años 1989 y 1995, y el artífice del resurgir del Museo tras años de olvido.

En esta reunión, un nutrido grupo de investigadores (ver programa en el Apéndice 1) de diferentes países teniendo en común su relación con Pere Alberch (cinco de ellos del Museo Nacional de Ciencias Naturales, entre los que me incluyo) han evocado la figura de éste en diferentes áreas, desde la museística a la investigadora, pasando por su interés en el diálogo arte-ciencia.

Tras una emotiva charla inaugural impartida por Jordi Alberch, hermano del homenajeado e investigador del Departamento de Biología Celular de la Facultad de Medicina de la Universidad de Barcelona, evocando los orígenes y la evolución de Pere desde una perspectiva familiar y científica, la conferencia plenaria a cargo del Profesor Adolf Seilacher versó acerca de la importancia de los procesos de desarrollo en la evolución de los sistemas de alimentación en Crinoideos, e introdujo los temas principales que se abordarían a lo largo de las diferentes sesiones relacionadas con las diferentes facetas e intereses del homenajeado.

Una sesión dedicada a Paleontología y Evolución incidió en la integración del estudio del desarrollo de los seres vivos y del registro fósil como uno de los pilares imprescindibles para desarrollar una teoría evolutiva completa. En ella se puso de mani- fiesto como las ideas de Pere acerca del desarrollo embrionario, como una fuerza que impone restricciones a las formas que pueden surgir a través del cambio evolutivo y como canalizador de éste, cobran sentido a la hora de estudiar diferentes problemas evolutivos con herramientas paleontológicas. Así, se habló de la aplicación del concepto de morfoespacio teórico (un espacio geométrico en el que coinciden las formas biológicas existentes y no existentes pero posibles) al estudio de la evolución en moluscos. También se habló de modelos en mosaico para la evolución del cráneo humano basados en análisis de cráneos de homínidos fósiles, de humanos de diferentes orígenes geográficos y en la existencia de módulos sometidos a restricciones diferentes durante el desarrollo del cráneo. Y se habló de la estabilidad fenotípica o conservación de las formas (en definitiva de la presencia de restricciones evolutivas) en la evolución de la pelvis de Archosaurus.

Otra sesión fue dedicada a Complejidad y Modelización, un tema en el que Pere estaba trabajando cuando la muerte le sorprendió. Es evidente, aunque muchas veces se ha ignorado, que los productos génicos, las células, los órganos y los organismos no existen aisladamente, sino que funcionan interaccionando con otros en redes complejas.

En esta sesión se exploró como a la hora de entender la complejidad de estas redes y el origen y evolución de las mismas es necesario integrar empirismo (observación y experimentación) y teoría, siendo un ejemplo de dicha integración el propio concepto de morfoespacio anteriormente citado. Durante la misma se presentó el modelo de redes mutualistas planta/ animal y la coevolución de organismos dentro de las mismas como un ejemplo de complejidad cuyo estudio es fundamental para

Dpto. Biodiversidad y Biología Evolutiva. Museo Nacional de Ciencias Naturales. CSIC. C/ José Gutierrez Abascal 2. 28006 Madrid. Tno: 914111328 Ext.1131. E-mail: mcnb@119mncn.csic.es 
entender la arquitectura de la biodiversidad. Asimismo, utilizando diferentes ejemplos de sistemas complejos (transcriptoma o maquinaria de trascripción del ADN, estructura del lenguaje, etc) se discutió como se origina y evoluciona esta complejidad. Finalmente quedó claro que para que dicha complejidad surja, se mantenga (robustez de las redes) y evolucione (evolubilidad -del inglés evolvability- de los sistemas) no es necesario ningún diseño inteligente. La propia dinámica de los sistemas desde su génesis garantiza estas propiedades.

Otra de las sesiones se dedicó a analizar las fuentes filosóficas de las que se nutrió el trabajo de Pere Alberch y otros que confluyeron en lo que hoy se conoce como EvoDevo (contracción de los términos ingleses para Evolución y Desarrollo), así como de las implicaciones de esta escuela en la propia filosofía de la ciencia. En ella se analizó el papel de la lógica matemática en el estudio de los fenómenos complejos o la influencia de la escuela morfogenética en el desarrollo de la propia EvoDevo. Se repasó el peso de lo inherente o propio de los sistemas biológicos frente al peso de las circunstancias históricas en que éstos existen (contingencia). Se habló de la fortaleza de los propios sistemas y como éstos aportan elementos para teorías más generales del conocimiento y se discutió sobre las formas sin función, las monstruosidades o teratologías a las que Pere dedicó algunos trabajos, como elementos para entender la organización de los procesos de desarrollo. En este sentido, simplemente decir que los monstruos naturales suponen una de las más claras evidencias de la existencia de restricciones impuestas por el desarrollo a la diferenciación de las formas. Así, por ejemplo, resulta evidente que cuando se origina una malformación durante el desarrollo embrionario de hombres o animales, solo algunas formas son posibles (dos cabezas pero no tres, siameses unidos por el tronco pero no por los pies, etc.).

Tres sesiones fueron dedicadas a la EvoDevo antes mencionada y uno de los campos en los que la figura de Pere Alberch alcanza su máxima dimensión científica. La EvoDevo introduce en la teoría evolutiva el papel del desarrollo como una fuerza interna capaz de imponer restricciones a las formas posibles y por tanto como un canalizador de la evolución, y en este sentido va un paso más allá que suponer que el proceso evolutivo es solamente modelado por la selección natural o la deriva genética como sostiene la visión imperante en evolución.

En estas sesiones se ha rememorado como surge esta escuela y cual es su situación en el panorama actual del pensamiento evolucionista analizándose las causas de que la EvoDevo todavía esté eclipsa- da por la visión predominante surgida de la Nueva Síntesis elaborada en los años treinta del siglo XX.

En el transcurso de éstas se ha analizado la contribución empírica de Pere Alberch en este campo, primero desde el laboratorio de David Wake en Berkeley (como el propio Wake expuso) estudiando la evolución y desarrollo de la extremidad de anfibios como modelo, trabajo que luego proseguiría en sus años en este Museo, y luego, ya instalado en Madrid, comenzando el estudio de la evolución de los modos de reproducción dentro de una única especie, Salamandra salamandra, estudios que han continuado algunos de sus discípulos.

Asimismo, se ha presentado una colección de ejemplos empíricos trabajando sobre diferentes sistemas biológicos que refuerzan la vitalidad de esta escuela y retan desde diferentes perspectivas la visión unitaria del proceso evolutivo como meramente modulado por selección y deriva.

Finalmente, una sesión estuvo dedicada a Museología y en ella se ha pasado revista al papel de Pere Alberch en este campo, sobre todo como renovador e impulsor de una nueva etapa del Museo Nacional de Ciencias Naturales tras años de olvido, así como a los retos de la Museología en este comienzo del siglo XXI, abordándose temas como la interacción entre instituciones, la gestión y política de las colecciones científicas que deben albergar los Museos, como hacer atractivas las exposiciones a través de la introducción de metáforas, o como se desarrollan y evolucionan los propios Museos, tomando como ejemplo a este Museo Nacional de Ciencias Naturales.

Clausuró esta reunión científica una charla del fotógrafo Joan Fontcuberta autor de las fotografías que sirvieron de base a la primera exposición montada durante la etapa de Pere Alberch como director del museo, "Fauna secreta", y un concierto en que música de jazz e imágenes de obras de arte y de procesos naturales dialogaron sobre la génesis de la forma.

Sin duda, a Pere le hubiera gustado este broche.

\section{AGRADECIMIENTOS}

El autor quiere agradecer a Antonio García Valdecasas sus sugerencias y comentarios en relación con el presente manuscrito. 
Apéndice 1. Program. Memorial Pere Alberch: The cradle of evo-devo. International Congress. Valencia 21-24 May 2008. Institute Cavanilles for Biodiversity and Evolutionary Biology, University of Valencia. Organizers: Diego Rasskin-Gutman, Miquel De Renzi, Andrés Moya \& Juli Peretó.

Welcome address

\section{Wednesday May 21}

19:00-19:30 Jordi Alberch

19:30-20:30 Adolf Seilacher.

Developmental Divergence in Pseudoplanktonic Crinoids

TAPAS PARTY

Thursday May 22

(1) Paleontology and Evolution 09:00-09:30. Intro: Miquel De Renzi

The work of Pere Alberch: developmental biology and evolutionary palaeobiology at the cross-roads

09:30-10:00. George McGhee

Evolutionary Development in Theoretical Morphospace 10:00-10:30. Antonio Rosas

Developmental aspects of mosaic evolution of the human skull

10:30-11:00. Angela Buscalioni

Insights on Phenotypic stability and von Baer statements

(2) Complexity AND Modeling

12:00:12:30. Intro: Diego Rasskin

Attractors in morphospace: Pere Alberch and DST in evo-devo

12:30-13:00. Jordi Bascompte

Plant-Animal Mutualistic Networks: The Architecture of Biodiversity

13:00-13:30. Ricard Solé

On networks, spandrels and monsters

13:30-14:00. Garret Odell

For making genetic networks operate robustly, unintelligent nondesign suffices

(3) Philosophy

16:00-16:30. Intro: Andrés Moya

When Logic reaches Biology: A historical precedent to approach the theory of complex biological phenomena

16:30-17:00. Werner Callebaut

Contingency and Inherency in Evo-Devo and Niche Construction

17:00-17:30. William Wimssatt

Pere Alberch, Developmental Constraints, and Generative Entrenchment

17:30-18:00. Arantza Etxeberria

Alberch on the role of forms without function to explain life
18:00-18:30. Laura Nuño de la Rosa

Pere Alberch and the Morphogenetic school in the history of EvoDevo

Friday May 23

(4) Developmental Biology \& Evolution I

09:00-09:30. Intro: Gerd Müller

EvoDevo then and now

09:30-10:00. Stuart Newman

Dynamical Patterning Modules: a "pattern language" for development and evolution of multicellular form

10:00-10:30. Adam Wilkins

Climbing Mount Improbable: why synthesizing EDB and evolutionary genetics is so difficult

\section{(4) Developmental Biology \& Evolution II}

12:00-12:30. Intro: David Wake

Pere Alberch and evodevo studies of amphibian limbs.

12:30-13:00. Alessandro Minelli

The evolving modes of evolution

13:00-13:30. Jorge Cubo

Towards a more integrated evolutionary morphology: Quantification of phylogenetic, functional and structural components of variation in bone growth rate of amniotes

13:30-14:00. James Hanken

Embryonic origin and segmentation of the amphibian skull: relation to a complex life history

(4) Developmental Biology \& Evolution III

16:00-16:30. Intro: Marvalee Wake

Pere Alberch and the Evolution of Viviparity

16:30-17:00. Adriá Casinos

Geoffroy Saint-Hilaire's loi du balancement revisited

17:00-17:30. Manuel Marí-Beffa

In the search of an actinoterygian ray-fin morphospace

17:30-18:00. Luis Boto

An overview in molecular mechanisms for acquisition of evolutionary novelties

(5) Museology

\section{Saturday May 24}

14:30-15:00. Intro: Jorge Wagensberg

Evolutionary biology in museology

15:00-15:30 Carme Prats, Rosa M. Malet

Opening the window: the Alberch legacy to museology. 1989-1995 


\section{5:30-16:00. Isabel Fuentes}

Visual metaphors that bring biology to museums

16:30-17:00. Intro: Margarita Belinchón

MNCN-Museo Paleontológico Rodrigo Botet: the role

of Pere Alberch in a symbiotic interaction between these two institutions

17:00-17:30. Borja Sanchiz

Developmental and evolutionary trajectories in Museology: the Museo Nacional de Ciencias Naturales as a case example

17:30-18:00. Antonio Valdecasas

Noah's 'take two of each' dilemma and museum collections
(6) Special Events, Arts and Nature 19:00-19:40 Joan Fontcuberta

Secret Fauna

19:45-20:30 Angela Buscalioni and Rafa Buscalioni

Closing performance: "The Genesis of Form".

20:30-21:00 Closing Remarks.

DINNER 